\title{
Simulating a digital business ecosystem
}

\author{
M. Petrou, S. Gautam \& K. N. Giannoutakis \\ Electrical and Electronic Engineering, Imperial College, \\ London, UK
}

\begin{abstract}
A digital business ecosystem (DBE) is a closed or semi-closed system of small and medium enterprises (SMEs), which will come together in cyberspace in the same way that companies gather in a business park in the physical world. These companies will interact with each other through buyer-seller relationships. The purpose of this work is to develop a methodology that will allow one to study the ecosystem under various conditions and we present here a model for the mutual interactions between companies in a DBE and a methodology that can allow one to study the dynamics of a digital business ecosystem. Furthermore we present a quantitative model for studying the dynamics of such a system, inspired by human physiology and attempting to capture many aspects of the way companies interact with each other, including the quantitative modelling of trust and mistrust.
\end{abstract}

\section{Introduction}

A digital business ecosystem (DBE) is a closed or semi-closed system of small and medium enterprises (SMEs), which will come together in cyberspace in the same way that companies gather in a business park in the physical world. These companies will interact with each other through buyer-seller relationships. The purpose of this work is to develop methodology that will allow one to study the ecosystem under various conditions. In particular, we would like to answer the following question: "Under the assumption that the ecosystem is closed, and static, ie no external influences, which of the companies in it are most likely to prosper and survive, and which are most likely to be suppressed?". This is a situation of competitive co-existence, where each unit receives excitatory and inhibitory signals from all other units in the system. Such systems exist in biology, and their states are known to oscillate between extremes, rather than to converge to a single 
steady state. The reason of these oscillations is the asymmetry with which they influence each other: A influences B in a different way by which B influences A. So Markov random fields are not appropriate for studying such a system because the interactions between the units are asymmetric. On the other hand, biological systems consist of neurons which interact with each other in a non-symmetric way $[11,12]$. Inspired from the work in $[11,12]$, we present here a model for the mutual interactions between companies in a DBE. This model yields a set of nonlinear coupled differential equations which in the case of $[4,11,12]$ govern the potential of each neuron in the visual cortex V1 and produce a saliency map of the viewed scene. In a DBE, instead of saliency of pixels we may have a fitness value of each company, or each product on sale. Following $[4,11,12]$ we solve the system of non-linear coupled differential equations which govern these fitness values as a neural network of nodes that exchange messages. In a biological system, the membrane potential of a neuron, which corresponds to its activation level, changes with time: the stronger it is, the faster it decays. So this membrane potential obeys a differential equation. For example, we know that whatever the potential of a neuron is, in lack of any external stimulus, it will decay with time exponentially:

$$
\frac{d y}{d t}=-\tau y \Rightarrow y=y_{0} e^{-\tau t}
$$

where $\tau$ is the time constant of the system, and $y_{0}$ is the value of $y$ for the boundary condition $t=0$.

In order to study the dynamics of an ecosystem, we must have first an instantiation of such a system. In Section 3 we show how we create a simulated DBE consisting of 100 companies which trade 20 products. The methodology we propose can be used to create realistic instantiations of a DBE, provided some statistical information is known from the real DBE we wish to study. In Section 4 we present the self-organising neural network we shall use for studying the dynamics of the simulated DBE. In Section 5 we present our experiments and results and we conclude in Section 6. We start, however, with a literature survey presented in Section 2.

\section{Literature survey}

There have not been many quantitative attempts to study DBEs. Most papers published follow the procedure of hypothesis generation, data collection by a survey or a questionnaire and finally hypothesis testing using statistical methods (e.g. $[5,6,13,16])$. There are various reasons for that: The complexity of the system, the multiplicity of the issues involved, and of course the lack of uniformity in the description of products and services, necessary to study the dynamics of a complex system [19]. The lack of such studies and the lack of quantitative measures that they could yield has consequences in the formation of economic policies for the internet [15]. We address the problem of lack of uniformity in this paper by created a realistic simulated DBE that shares its statistical properties with a real DBE. 
In spite of the above mentioned difficulties, some attempts to quantify at least some of the relevant quantities in an e-commerse system have already been made. For example, Manchala in [14] makes a serious attempt to quantify trust by counting the number of transactions a vendor feels the need to verify before they proceed with the actual transaction. Manchala stresses the need for quantitative measures of trust in order to make quantitative studies of such systems. In this paper we quantify trust by invoking the psychophysical law of Weber Fechner (see Section 3). Our approach is not incompatible with the approach of Manchala: he starts from some objective measure; we start from qualitative categories of trust and try to infer from them some objective rankings. In a sense, if people were asked to use the quantitative measure of Manchala to create categories of trust, we believe that they would create categories that could be modelled by the psychophysical law of Weber Fechner. We believe that this law can bridge the gap between models like the one presented in [8], which uses qualitative terms like "low risk", "high risk" etc, and more quantitative studies like the one in [20]. Another attempt to use a quantitative model is that of Cheung and Liao [2] who produce a quantitative measure of shoppers' willingness to buy. The model is a simple regression formula, where the independent variables are the statistical scores of acceptance or rejection of certain hypotheses tested by surveys.

The importance of trust on web based transactions has been stressed by many researchers [10], to the point that there are even papers on how to build web-based systems that inspire trust to the customer $[1,16,17]$. Other people have studied the effect of trust by looking at the way web-sites evolve over time, their structure and of course by conducting surveys [18].

Most of the studies, qualitative or quantitative, concentrate on the binary interaction between supplier and buyer. One of the first attempts to try to model higher order interactions in a business environment is the one presented in [3]. This model, however, is still qualitative.

Our methodology of producing simulated DBEs may also allow the testing under controlled realistic conditions, of algorithms designed to work with real data, like for example the algorithm of Sung et al. [20] designed to cluster products according to their attributes in order to create product catalogues. Simulation experiments for studying on line stores are not novel. For example Gefen et al. used the model presented in [5] in a simulated environment to study the effect of trust using simulated scenaria.

\section{A simulated DBE}

Here we present methodology on how to construct a realistic simulated DBE, based on observations of a real DBE. We developed a software package which creates a database of companies and products that have the same statistical properties as in the observed DBE. This program has the flexibility to create a database of any number of companies and products. Each company created is assigned a SELL and a WANT list. The SELL list of a company is the list of the products the company wants to sell and the WANT list of a company is the list of products the company 
wants to buy. Either of these lists might be empty, but not both. All products which appear in the SELL list of all companies make up the database of real products. All products which appear in the WANT lists of all companies make up the database of virtual products, because these products exist in the customers' minds. A product may appear in both databases, but it most likely will have different attributes in the two databases. A product has the same name, type and number of attributes no matter in which of the two databases it appears. What changes from one database to the other is the statistics of the attributes which characterise each product. Two types of attribute are catered for, numerical and symbolic. The statistics of the numeric attributes are characterised by their mean and standard deviation, which are assumed to be extracted by observing a real DBE. Each symbolic attribute takes values from a list of symbols, with probabilities according to the frequency with which each such value is encountered in the real DBE.

In addition, each company is assigned two other lists: the TRUST list which contains the names of the other companies in the ecosystem that it trusts, and the MISTRUST list which contains the names of the companies that it mistrusts. Any company that does not appear in either of the two lists is unknown to the company in terms of trustworthiness. We also model the effect of the spreading reputation of each company for these lists. When populating the TRUST or MISTRUST list of a company, we gave an extra weight to those companies which had already appeared in already created corresponding lists of other companies. To model the fact that good reputation propagates faster than bad reputation, the weights used for the TRUST lists are higher than the weights used for the MISTRUST lists.

Finally, we propose to use the psychophysical law of Weber-Fechner in order to convert the qualitative concepts of trust, indifference and mistrust to numerical weights for the case one wishes to construct numerical models to study these factors. The idea is to use this law to go from subjective classes of trust to relative numerical measurements that somehow reflect objectivity. According to this law, the degree of subjective judgement is proportional to the logarithm of an objective measure that measures the same effect. For example, if in your mind you create categories of untrustworthiness and you call them 1,2 and 3, the people whom you classify in these categories have to lie to you twice, four times or eight times, respectively, for you to put them in the respective categories. So, we argue that categories of untrusted, indifferent and trusty correspond to some arbitrary objective numerical values proportional to 2,4 and 8 , respectively. As these values have to be used to weigh relatively the various possible transaction partners, their exact values do not matter. To make them into relative weights, these values are normalised to sum to 1 , so in the model we shall present in the next section we shall use weights $\frac{2}{14}=0.14, \frac{4}{14}=0.29$ and $\frac{8}{14}=0.57$ for undesirable, indifferent and desirable partner respectively.

\section{Modelling the competitive co-existence of companies}

Let us assume that each company $C_{i}$ has with it associated a positive variable, $y_{i}$, which measures how well the company does and how strong it is, and let us call 
it the fitness variable. This is an abstract quantity with no units associated with it, and it should not be confused with economic indicators like cash flow, volume of transactions etc. If the company is left on its own, in isolation, the value of $y_{i}$ will decay according to equation (1) because a company of course cannot exist in isolation and with no interactions with other companies/customers. For simplicity, let us assume that for all companies, the decaying constant $\tau$ is the same. First we shall produce a simple model for variable $y_{i}$. The differential equation obeyed by $y_{i}$ will have to model the following effects, yielding extra terms that have to be included on the right-hand-side of equation (1):

- The stronger a company is, the more strong it is likely to become. This is a self-excitation term, of the form $J_{0} g_{y}\left(y_{i}\right)$. Self-excitation constant $J_{0}$ again is assumed to be the same for all companies. Function $g_{y}\left(y_{i}\right)$ is a sigmoid function: effects in real life are only linear over a certain scale. They saturate and the benefit we receive by changing the independent variable $y_{i}$ levels off. On the other hand, before this positive feedback in the strength is triggered, a so called "critical mass" of strength $y_{i}$ has to be reached. So, function $g_{y}\left(y_{i}\right)$ may be modelled as:

$$
g_{y}\left(y_{i}\right)= \begin{cases}0 & \text { if } y_{i}<\Gamma_{1}, \\ \frac{\left(y_{i}-\Gamma_{1}\right)}{\left(\Gamma_{2}-\Gamma_{1}\right)} & \text { if } \Gamma_{1} \leq y_{i} \leq \Gamma_{2} \\ 1 & \text { if } y_{i}>\Gamma_{2}\end{cases}
$$

where $\left[\Gamma_{1}, \Gamma_{2}\right]$ is the range of linearity of the positive gain function.

- A term that models all excitatory signals the company receives from all other companies. First we have to quantify the excitatory signal a company $C_{i}$ receives from another company $C_{j}$. A company will stimulate another company if they demand products that match those the other company sells. Let us say that one of the products a company $C_{i}$ wishes to buy is product $P$, with attributes $x_{l}^{P}$ for $l=1,2, \ldots, L_{P}$, with $L_{P}$ being the number of attributes that characterise product $P$. There may be several companies $C_{j}$ in the ecosystem that provide product $P$ with attributes similar to those requested. The mismatch value of product $P$ between the attributes company $C_{i}$ requires and those of the same product company $C_{j}$ sells may be computed as

$$
V_{P i j} \equiv \sum_{l=1}^{L_{P}} w_{P l} V_{l P i j}
$$

where if attribute $l$ is numeric

$$
V_{l P i j} \equiv \frac{\left|x_{l}^{P j}-x_{l}^{P i}\right|}{x_{l}^{P i}}
$$


and if attribute $l$ is symbolic:

$$
V_{l P i j} \equiv \begin{cases}0 & \text { if } x_{l}^{P j}=x_{l}^{P i} \\ 1 & \text { if } x_{l}^{P j} \neq x_{l}^{P i}\end{cases}
$$

$V_{P i j}$ is the mismatch value of attribute $l$, between the required product by company $i$ and the corresponding product supplied by company $j$. The weights $w_{P l}$ express the relative importance for each attribute. They are normalised so that they sum up to 1 . If $V_{P i j}$ is below a certain threshold $T_{1}$, we may assume that the products match. The more such products match, the more likely it is that company $C_{i}$ will receive positive stimulation from company $C_{j}$. Let us say, therefore, that we count all products $P$ which appear in the WANT list of company $C_{i}$ and in the SELL list of company $C_{j}$ and for which $V_{l P i j} \leq T_{1}$ and find them to be $E_{i j}$. We may define then the excitatory signal $C_{i}$ may receive from $C_{j}$ as

$$
J_{i j} \equiv 1-e^{-E_{i j}}
$$

Note that the higher $E_{i j}$ is, the more $J_{i j}$ will tend to 1 , while when $E_{i j}=0$, ie when no products match, $J_{i j}=0$ too. Also note that the excitatory signal company $C_{j}$ sends to $C_{i}$ is not the same as the excitatory signal $C_{i}$ sends to $C_{j}$. In other words $E_{i j} \neq E_{j i}$, as $E_{j i}$ will count the pairs of products that are less dissimilar than $T_{1}$ from the sets of the WANT list of company $C_{j}$ and the SELL list of company $C_{i}$. In addition, we must also realise that a company $C_{j}$ will stimulate company $C_{i}$ only if $C_{j}$ is healthy and strong itself. A company that is very weak will probably not create much volume of trading. So, the excitatory signal $J_{i j}$ must be modulated by $g_{y}\left(y_{j}\right)$ to account for that. In addition, company $C_{i}$ will trade with company $C_{j}$ only if it trusts it. So, this excitatory signal should also be weighed by the trust company $C_{i}$ has to company $C_{j}$. This appears as a factor $W_{i j}$, which takes values $\frac{4}{7}, \frac{2}{7}, \frac{1}{7}$ when company $C_{j}$ is trusted, is indifferent or mistrusted by company $C_{i}$, respectively. Finally, we must sum up all such positive influences $C_{i}$ receives from all other companies in the ecosystem. So, the term we must add on the right-hand-side of (1) should be:

$$
\sum_{j \in C, j \neq i} W_{i j} J_{i j} g_{y}\left(y_{j}\right)
$$

- A term that models all inhibitory signals the company receives from all other companies. First we have to quantify the inhibitory signal a company $C_{i}$ receives from another company $C_{j}$. A company will inhibit another company if both companies sell similar products. So, first we need to quantify the dissimilarity between a product $P$ both companies sell. To do that we use equation:

$$
U_{P i j} \equiv \sum_{l=1}^{L_{P}} w_{P l} U_{l P i j}
$$


where if attribute $l$ is numeric

$$
U_{l P i j} \equiv \frac{\left|x_{l}^{P j}-x_{l}^{P i}\right|}{x_{l}^{P i}}
$$

and if attribute $l$ is symbolic

$$
U_{l P i j} \equiv \begin{cases}0 & \text { if } x_{l}^{P j} \equiv x_{l}^{P i} \\ 1 & \text { if } x_{l}^{P j} \neq x_{l}^{P i}\end{cases}
$$

$U_{P i j}$ measures the dissimilarity between product $P$ companies $C_{i}$ and $C_{j}$ sell. If this number is below a certain threshold $T_{2}$, we may assume that the products match. The more such products match, the more likely it is that company $C_{i}$ will receive inhibitory signals from company $C_{j}$. Let us say, therefore, that we count all products that appear in the SELL lists of both companies for which $U_{P i j} \leq T_{2}$ and find them to be $F_{i j}$. We may define then the inhibitory signal $C_{i}$ receives from $C_{j}$ as

$$
K_{i j} \equiv 1-e^{-F_{i j}}
$$

Note that the higher $F_{i j}$ is, the more $K_{i j}$ will tend to 1 , while as $F_{i j} \rightarrow 0$, $K_{i j} \rightarrow 0$ too. We note that $F_{i j}=F_{j i}$, as $F_{j i}$ will count the pairs of products that are less dissimilar than $T_{2}$ sold by both companies. In addition, we must also realise that a company $C_{j}$ will inhibit company $C_{i}$ only if $C_{j}$ is healthy and strong itself. So, the inhibitory signal $K_{i j}$ must be modulated by $g_{y}\left(y_{j}\right)$ to account for that. Finally, we must sum up all such negative influences $C_{i}$ receives from all other companies in the ecosystem. So, the term we must add on the right-hand-side of (1) should be:

$$
-\sum_{j \in C, j \neq i} K_{i j} g_{y}\left(y_{j}\right)
$$

- We may also include a term which may be external input to the company, like total volume of transactions originating outside the DBE, or something like that, properly scaled to be a dimensionless number. Let us call this $I_{i}$.

- Finally, we may add a term that expresses the background input, eg the general economic climate, and it is the same for all companies in the ecosystem. Let us call it $I_{0}$.

If we put all the above together, we come up with the following differential equation that has to be obeyed by the fitness variable of company $C_{i}$ :

$$
\frac{d y_{i}}{d t}=-\tau_{y} y_{i}+J_{0} g_{y}\left(y_{i}\right)+\sum_{j \in C, j \neq i} W_{i j} J_{i j} g_{y}\left(y_{j}\right)-\sum_{j \in C, j \neq i} K_{i j} g_{y}\left(y_{j}\right)+I_{i}+I_{0}
$$

This is a set of coupled differential equations concerning all companies in the ecosystem. If we solve it, we may be able to see the combination of values of the 
fitness variables that will tell us which companies will dominate the ecosystem. Equation (13) may be solved as difference equations applied to the nodes of a fully connected network, the values of which are updated in an iterative scheme.

$$
\begin{aligned}
y_{i ; \text { new }}-y_{i ; \text { old }}= & -\tau_{y} y_{i ; \text { old }}+J_{0} g_{y}\left(y_{i ; \text { old }}\right)+ \\
& \left\{\sum_{j \in C, j \neq i} W_{i j} J_{i j} g_{y}\left(y_{j}\right)-\sum_{j \in C, j \neq i} K_{i j} g_{y}\left(y_{j}\right)\right\}_{\text {old }}+I_{i}+I_{0}
\end{aligned}
$$

The values of $y_{i}$ are initialised to be all equal to 1 at the first step. After each update cycle, we may remove from the system the companies the fitness value of which is below a certain threshold $T_{3}$. At the same time, we may allow the introduction of new companies with a certain rate, giving them as starting fitness value the average fitness of all other companies. In the next section, this model is investigated for various values of its fixed parameters, in order to observe the behaviour of the system under different conditions.

\section{Some experimental results}

We have started a series of extensive experiments in order to study the effect of each one of the parameters of the system to the dynamics of the system. The input data are the simulated DBE we constructed in Section 3. Some preliminary results are presented here. Figure 1 shows the number of companies that survive as a function of the number of iterations the system is allowed to run, for certain parameter values. In all these experiments, the following parameter values were used: $J_{0}=I-i=I_{0}=T_{1}=T_{2}=T_{3}=0.2, \Gamma_{1}=0.5$ and $\Gamma_{2}=1.5$. Figure 2 shows the fitness values of the various companies after 7 and 12 iterations when a monopoly was created. The parameter values that resulted in the monopoly were $\tau=2.0, \Gamma_{1}=0.5, \Gamma_{2}=1.5, J_{0}=I_{i}=I_{0}=2.5$ and $T_{1}=T_{2}=T_{3}=0.2$.

\section{Discussion and conclusions}

We presented here methodology that can allow one to study the dynamics of a digital business ecosystem. Such systems tend to be distributed in cyberspace and it is not possible to have real data for them. However, one may relatively easily acquire statistical data by using for example, a web robot, or another program designed for the purpose. The idea then is to use the gathered statistical data to produce a simulated version of the DBE which shares the same statistical properties as the real DBE. Such methodology has been used for many years by scientists to study complex systems that cannot be modelled in a deterministic way. For example, astronomers have learnt a lot about the dynamics of galaxies by studying simulated models of them.

Further, we presented a quantitative model for studying the dynamics of such a system, inspired by human physiology and attempting to capture many aspects of 


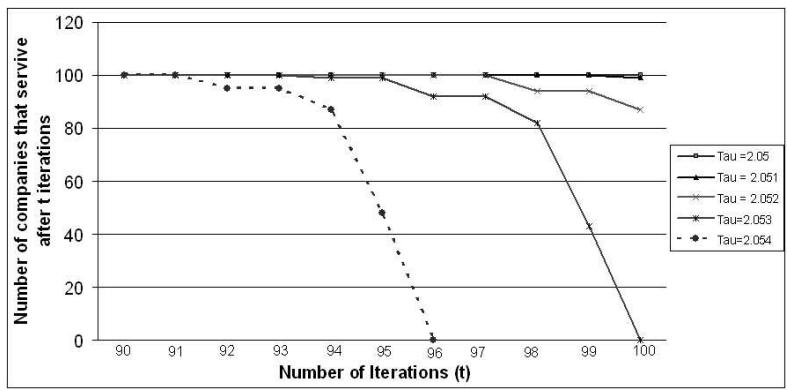

Figure 1: Number of companies that survive as a function of iterations, for various values of parameter $\tau$ and the remaining parameters fixed to $J_{0}=I_{i}=$ $I_{0}=T_{1}=T_{2}=T_{3}=0.2$.
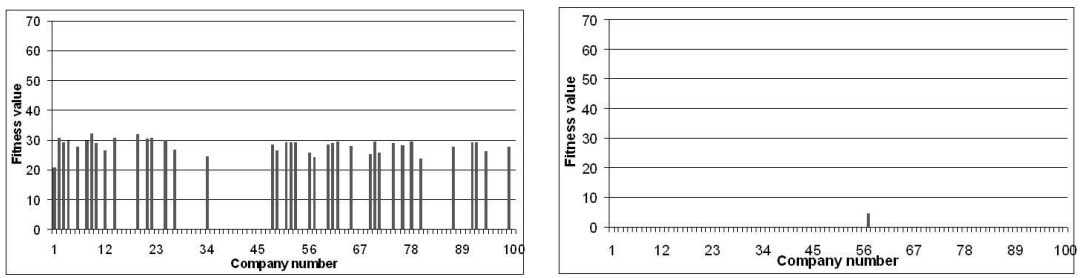

Figure 2: Monopoly created after 12 iterations. The fitness values of the companies after 7 and 12 iterations.

the way companies interact with each other, including the quantitative modelling of trust and mistrust. Several improvements to the model can be made. For example, one refinement one may make concerns the modelling of the mutual inhibition of two companies: At the moment we model this taking into consideration only the products both companies try to sell. This is fine in an open environment where the supply is infinite. However, in a closed environment when the supply is finite, two companies may exchange inhibitory signals even when they simply want to buy the same product or service. In this case we shall have to modify the calculation of term $K_{i j}$ to rely also on the common products two companies seek to purchase. Other improvements will involve the injection of new companies into the system, in a random way.

Of course, the final step to make such a model really useful would be to be able to associate the values of its various parameters with real measurable values from the observed DBE. At this stage, only the values of the parameters that control the creation of the simulated DBE, according to Section 3, can be directly associated with measurable quantities. The values of the parameters used to study its dynamics, according to the model of Section 4, have also to be associated with real measurable quantities. This is an important big task on its own. 


\section{References}

[1] Cook, D.P. \& Luo, W., The role of third-party seals in building trust on line, e-Service Journal, Indiana University Press, 2003.

[2] Cheung, M.T. \& Liao, Z., Supply-side hurdles in internet B2C e-commerce: an empirical investigation, IEEE Transactions on Engineering Management, 50(4), pp. 458-469.

[3] Choi, T.Y., Wu, Z., Ellram, L. \& Koka, B.R., Supplier-supplier relationships and their implications for buyer-supplier relationships, IEEE Transactions on Engineering Management, 49(2), pp. 119-130, 2002.

[4] Dayan, P. \& Abbott, L.F., Theoretical neuroscience:computational and mathematical modelling of neural systems, 2001.

[5] Gefen, D., E-commerce: the role of familiarity and trust, Omega, 28, pp. 725-737, 2000.

[6] Gefen, D. \& Straub, D., Managing user-trust in B2C e-services, e-Service Journal, Indiana University Press, 2003.

[7] Gefen, D., Karahanna, E. \& Straub, D.W., Inexperience and experience with online stores: the importance of TAM and trust, IEEE Transactions on Engineering Management, 50(3), pp. 307-321, 2003.

[8] Jahng, J., Jain, H. \& Ramamurthy, K., Effective design of electronic commerce environments: a proposed theory of congruence and an illustration, IEEE Transactions on System, Man and Cybernetics, Part A: Systems and Humans, 30(4), pp. 456-471, 2000.

[9] Jayaraman, V. \& Baker, T., The internet as an enabler for dynamic pricing of goods, IEEE Transactions on Engineering Management, 50(4), pp. 470-477, 2003.

[10] Komiak, S.Y.X., Wang, W. \& Benbasat, I., Trust building in virtual salespersons versus in human salespersons; similarities and differences, eService Journal, Indiana University Press, 2005.

[11] Li, Z., Visual segmentation by contextual influences via intra-cortical interactions in the primary visual cortex, Networks:Computation in Neural Systems 10 pp. 187-212, 1999.

[12] Li, Z., Computational design and nonlinear dynamics of a recurrent network model of the primary visual cortex, Neural Computation, 13(8), pp. 17491780, 2001.

[13] Limayem, M., Khalifa, M. \& Frini, A., What makes consumers buy from internet? A longitudinal study of online shopping, IEEE Transactions on System, Man and Cybernetics, Part A: Systems and Humans, 30(4), pp. 421432, 2000.

[14] Manchala, D.W., E-commerce trust metrics and models, IEEE Internet Computing, pp. 36-44, March-April 2000.

[15] McKnight, L.W. \& Bailey, J.P., Internet Economics: when constituencies collide in cyberspace, IEEE Internet Computing, pp. 30-37, NovemberDecember 1997. 
[16] Noteberg, A., Christiaanse, E. \& Wallage, P., Consumer Trust in electronic channels, e-Service Journal, Indiana University Press, 2003.

[17] Patrick, A.S., Building trustworthy software agents, IEEE Internet Computing, pp. 46-52, November-December 2002.

[18] Ruppel, C., Underwood-Queen, L. \& Harrington, S.J., e-Commerce: The roles of Trust, security and type of e-commerce involvement, e-Service Journal, Indiana University Press, 2003.

[19] Singh, M.P., The e-commerce inversion, IEEE Internet Computing, pp. 4-5, September-October 1999.

[20] Sung, W.K., Yang, D., Yiu, S.M., Cheung, D.W., Ho, W.S. \& Lam, T.W., Automatic Construction of online catalog topologies, IEEE Transactions on System, Man and Cybernetics, Part C: Applications and Reviews, 32(4), pp. 382-391, 2002. 